\title{
Patient Demographics and Characteristics of Infection with Carbapenem-Resistant Acinetobacter baumannii in a Teaching Hospital from the United States
}

\author{
Nachiket D. Vaze, Christopher L. Emery, Richard J. Hamilton, Ari D. Brooks, Suresh G. Joshi\# \\ Surgical \& Nosocomial Infection Research and Bacterial Pathogenesis Program, Drexel University College of Medicine, Hahnemann \\ University Hospital, Philadelphia, USA \\ Email: "Suresh.Joshi@drexelmed.edu
}

Received November $13^{\text {th }}, 2012$; revised December $15^{\text {th }}, 2012$; accepted January $18^{\text {th }}, 2013$

\begin{abstract}
A characterization of the clinical demographic features of patients with infection caused Acinetobacter baumannii, and the antibiotyping of the isolates recovered from these patients was undertaken, with a special reference to carbapenemresistant variants, and their risk factors. This study was conducted retrospectively from January 2010 to March 2011 at a 616-bed tertiary care university hospital. Sixty-four patients were identified. Clinical and microbiological data were analyzed for risk factors and demographic features to derive relative risk and odds ratio. We identified 100 A. baumannii from 64 patients during 15 months period. Significant risk factors were working age (18 - 60 years), male gender, hospital stay ( $>1$ week but $<1$ month), prior hospitalization, in a progressive care nursing units, respiratory/mechanical ventilation, polymicrobial infections and prior antibiotic therapy. Methicillin-resistant staphylococcus aureus (MRSA), Pseudomonas aeruginosa, and Klebsiella pneumoniae were the significant co-infecting agents. The antibiogram demonstrated multidrug resistance in a majority of the isolates. Relative risk associated with ventilator, diabetes, and surgery was higher in repeat isolates than in first isolates and were multidrug resistant. Repeat isolates were more resistant that the first isolates to most anti-acinetobacter agents, but the overall crude mortality was not significant during this study period, and couldn't correlated to the choice of treatment. In conclusion, a resistance against all clinically used carbapenems, and colistin is rapidly increasing in repeat isolates of A. baumannii; leaves narrow therapeutic options to treat multidrug-resistant and pandrug-resistant $A$. baumannii infection. For the first time we report rising incidence of colistin resistance by 20 percent in repeat isolates, and is worrisome for healthcare centers. A combination therapy should be adopted to treat such infection to avoid the emergence of colistin-resistant phenotypes in the United States.
\end{abstract}

Keywords: Acinetobacter baumannii; Carbapenem Resistance; Colistin, Imipenem; Meropenem; Multidrug Resistance; Risk Factor

\section{Introduction}

Acinetobacter baumannii is a third most prevalent nosocomial pathogenic species. It has been isolated from various hospital environments and has been shown to colonize patients. Until recently, this pathogen was found primarily outside the United States, mainly in Europe and Asia [1,2], but it is emerging in the United States [3]. In addition, A. baumannii infections have been identified in troops returning from military engagements in the Middle East [4]. A. baumannii has been identified as the causative agent in many serious medical conditions such as sepsis and nosocomial pneumonia [5,6]. Although in general the virulence of this species is low; its colonization poses a threat in a health care setting. Pneumonia

\footnotetext{
*All authors report no conflict of interest related to this manuscript.

${ }^{\#}$ Corresponding author.
}

caused by A. baumannii is associated with contaminated or colonized surgical and respiratory equipment. Outbreaks of A. baumannii have been reported in hospitals; especially in intensive care units (ICUs) [7]. Its status as an opportunistic organism makes it an unpredictable nosocomial pathogen that can cause significant mortality in such cases [8-10].

A. baumannii is inherently resistant to many antimicrobials and can further acquire resistance to additional antimicrobials to become multidrug resistant (MDR). MDR- A. baumannii is a major cause of concern in health care settings, and the infections are extremely difficult to treat $[11,12]$. MDR- A. baumannii is usually treated with broad-spectrum antibiotics, including carbapenems. Carbapenems such as imipenem and meropenem are often the antibiotics of last resort for such infection, and 
resistance against them is considered an alarming situation $[13,14]$. Many risk factors are associated with the prevalence of A. baumannii and the development of MDRA. baumannii [15]. Environmental risk factors such as intubation [16], prior surgery [17], and length of stay in the ICU [18] can lead to the development of MDR in A. baumannii.

In this study, the sensitivity of the isolates to imipenem and meropenem was determined; imipenem-resistant and meropenem-resistant isolates were further analyzed for the presence of risk factors. One of the major reasons for antibiotic resistance is the persistence of infection and recolonization with the variants that can lead to repeat isolation of the same strain or a related strain [19]. This phenomenon was also analyzed in the present study. The repeat isolates are often much more virulent and more likely to be MDR [20,21]. Because obtaining repeat isolates is specifically related to hospital environments and therapy, a risk analysis was performed on these isolates for resistance to imipenem and meropenem.

\section{Materials, Methodology, and Set up}

The study was conducted during Jun 2010 through Dec 2011 at a 616 bed tertiary care university hospital. Patients with Acinetobacter-positive cultures were identified from the microbiology laboratory database, and the cases of $A$. baumannii infection were considered. The isolates were identified using standard microbiological laboratory tests and the API 20E and VITEK systems (bioMérieux, Durham, NC). Antibiotic susceptibility data were obtained using the VITEK 2 system (bioMérieux) and minimum inhibitory concentrations (MICs) carried out using agar microdilution technique, following the Clinical and Laboratory Standards Institute (CLSI) guidelines. For each patient and each sample, clinical data were accessed through the hospital online patient database. The criterion for infection versus colonization was interpreted as per Center for Disease Control and Prevention (CDC) guidelines [22]. The isolate was considered as multidrug resistant, if resistant to more than two classes of antimicrobials or to carbapenems. The first isolate was defined as the organism that was first isolated from the sample during hospital admission and the repeat isolate as the one that was obtained from the same patient after the isolation of the first isolate (usually after an interval of a week). Statistical analysis was carried out using GraphPad version 3.0 (GraphPad Software, LaJolla, CA). Fisher's exact test was applied, and odds ratios (OR) and risk ratios (RR) with $95 \%$ confidence interval (CI) values were calculated. A $P$ value $<0.05$ was considered significant.

\section{Results}

Total 100 isolates were recovered from 64 cases of infection with A. baumannii were considered, having distinct episodes of hospitalization. Table $\mathbf{1}$ demonstrates analysis of the demographic features. The patients were grouped into two broad categories according to age to enable comparison for analysis. Patients of working age (18 - 60 years) had a significantly higher chance of $A$. baumannii infection than seniors (65 and older). More men than women had infections with $A$. baumannii $(\mathrm{OR}=2.78$; CI $=1.36-5.68 ; P=0.0078)$. There was significant association between a stay in the hospital of less than 1 month but longer than 1 week and isolation of $A$. baumannii compared to a longer stay in the hospital (OR $=3.51$, CI $=1.71-7.20 ; P=0.0009)$. However, there was no significant correlation between the samples collected during the hospital stay, and culture positivity in regard to relative risk.

A source of sample is the important epidemiological indicator. Almost half $(49 \%)$ of the isolates were from sputum and tracheal secretion; $36(73 \%)$ of the patients with $A$. baumannii isolated from sputum were on a mechanical ventilator. Sputum was a more likely source of A. baumannii infection than bronchial wash $(\mathrm{OR}=12.76$, $\mathrm{CI}=5.39-30.24 ; P=0.0001)$. Most of the samples collected from ventilated patients had at least one other bacterium. The most prevalent co-isolated organisms were $P$. aeruginosa $(25 \%)$ and methicillin-resistant S. aureus $(19 \%)$ (Data is not shown).

The isolates were tested using the VITEK 2 system for susceptibility against commonly used antibiotics and categorized as resistant and susceptible groups according to the results, interpreted as per the CLSI criteria. Figure 1 is a graphical presentation of antibiogram using common antimicrobial agents, and indicates high levels of resistance across drug classes. More than $50 \%$ of the isolates were resistant to seven different antibiotics. Levofloxacin was the most ineffective antibiotic. Even a combination of piperacillin-tazobactam was ineffective. Both imipenem and meropenem showed resistance to $44 \%$ and $49 \%$ of the isolates, respectively. The only antibiotics that were relatively effective against $A$. baumannii infections were amikacin and colistin, and showed $30 \%$ and $11 \%$ of the isolates as resistant phenotypes (respectively), during studies of first isolates. Total 36 repeat isolates were included in this study. Some patients had no repeat isolates whereas some cases had more than two. A separate analysis was performed on these two groups, and their antibiogram and risk factor analysis were compared. The comparison demonstrates an increase in resistance in the repeat isolates against almost all antibiotics tested (Figure 1). The levels of resistance have increased by $30 \%$ for levofloxacin, by $32 \%$ for trimethoprim-sulfamethoxa- 
Table 1. Clinical demographic characteristics of Acinetobacter baumannii infections.

\begin{tabular}{|c|c|c|c|c|c|c|c|}
\hline Characteristic & $\begin{array}{c}\text { Number of patients } \\
(\mathrm{n}=64)\end{array}$ & Total (\%) & Groups to compare & OR & $95 \%$ CI & $\mathbf{R R}$ & $P$ value \\
\hline \multicolumn{8}{|l|}{ Age } \\
\hline 18 to 40 years & 18 & 28 & Working group vs seniors & 6.01 & $2.82-13.0$ & 2.5 & 0.0001 \\
\hline 41 to 64 years & 27 & 42 & & & & & \\
\hline 65 years and older & 18 & 28 & & & & & \\
\hline Infant & 1 & 3 & & & & & \\
\hline \multicolumn{8}{|l|}{ Gender } \\
\hline Female & 24 & 37 & & & & & \\
\hline Male & 40 & 63 & Male vs female & 2.78 & $1.36-5.68$ & 1.67 & 0.0078 \\
\hline \multicolumn{8}{|l|}{ Duration of hospital stay } \\
\hline Same-day discharge & 4 & 6 & & & & & \\
\hline 1 week & 15 & 23 & & & & & \\
\hline $\begin{array}{l}\text { More than } 1 \text { week but less than } 1 \\
\text { month }\end{array}$ & 26 & 39 & & & & & \\
\hline More than 1 month & 21 & 32 & $<1$ month vs $>1$ month & 3.51 & $1.71-7.20$ & 1.95 & 0.0009 \\
\hline Characteristic & $\begin{array}{l}\text { Number of cases } \\
\quad(\mathbf{n}=100)\end{array}$ & Total (\%) & & OR & CI & $\mathbf{R R}$ & $P$ value \\
\hline \multicolumn{8}{|l|}{ Time to culture of samples } \\
\hline Day of admission & 29 & 29 & & & & & \\
\hline Less than 1 week of hospitalization & 22 & 22 & & & & & \\
\hline $\begin{array}{l}\text { More than } 1 \text { week but less than } 1 \\
\text { month }\end{array}$ & 31 & 31 & & & & & \\
\hline More than 1 month & 18 & 18 & & & & & \\
\hline \multicolumn{8}{|l|}{ Source of sample } \\
\hline Anerobe & 9 & 9 & & & & & \\
\hline Blood & 10 & 10 & Sputum vs blood & 8.65 & $4.03-18.53$ & 4.9 & 0.0001 \\
\hline Bronchi & 7 & 7 & & & & & \\
\hline Tissue & 1 & 1 & & & & & \\
\hline Urine & 13 & 13 & & & & & \\
\hline Wound & 11 & 11 & Sputum vs wound & 7.77 & $3.71-16.28$ & 4.45 & 0.0001 \\
\hline Sputum & 49 & 49 & Sputum vs bronchial wash & 12.76 & $5.39-30.24$ & 7 & 0.0001 \\
\hline
\end{tabular}

oxazole (Bactrim), and colistin to $22 \%$. There was an increase in the levels of resistance to imipenem $(44 \%$ to $77 \%$ ) and meropenem (49\% to $71 \%)$.

Table 2 demonstrates the risk factor analysis for resistance to carbapenem. Risk factors for resistant isolates were determined through clinical data analysis, and the odds ratios were calculated. Prior hospitalization was a major risk factor for development of resistance to both drugs. Significantly higher odds of developing a resistant isolate were observed for carbapenem $(\mathrm{OR}=5.56, \mathrm{CI}=$
2.7 - 11.47; $P \leq 0.05$ ). Samples from the ventilated patients had an odds ratio of 1.9 for resistance to carbapenem $(\mathrm{OR}=1.9, \mathrm{CI}=1.01-3.52 ; P \leq 0.05)$. A greater number of resistant samples were taken from patients who were hospitalized for less than a month compared to patients with longer hospital stays $(\mathrm{OR}=3.58, \mathrm{CI}=1.78$ - 7.2; $P \leq 0.05)$. Sputum samples did not have a statistically significant risk for development of resistance to either carbapenem. Diabetes was not associated with risk of resistance in this analysis. Carbapenem here includes 


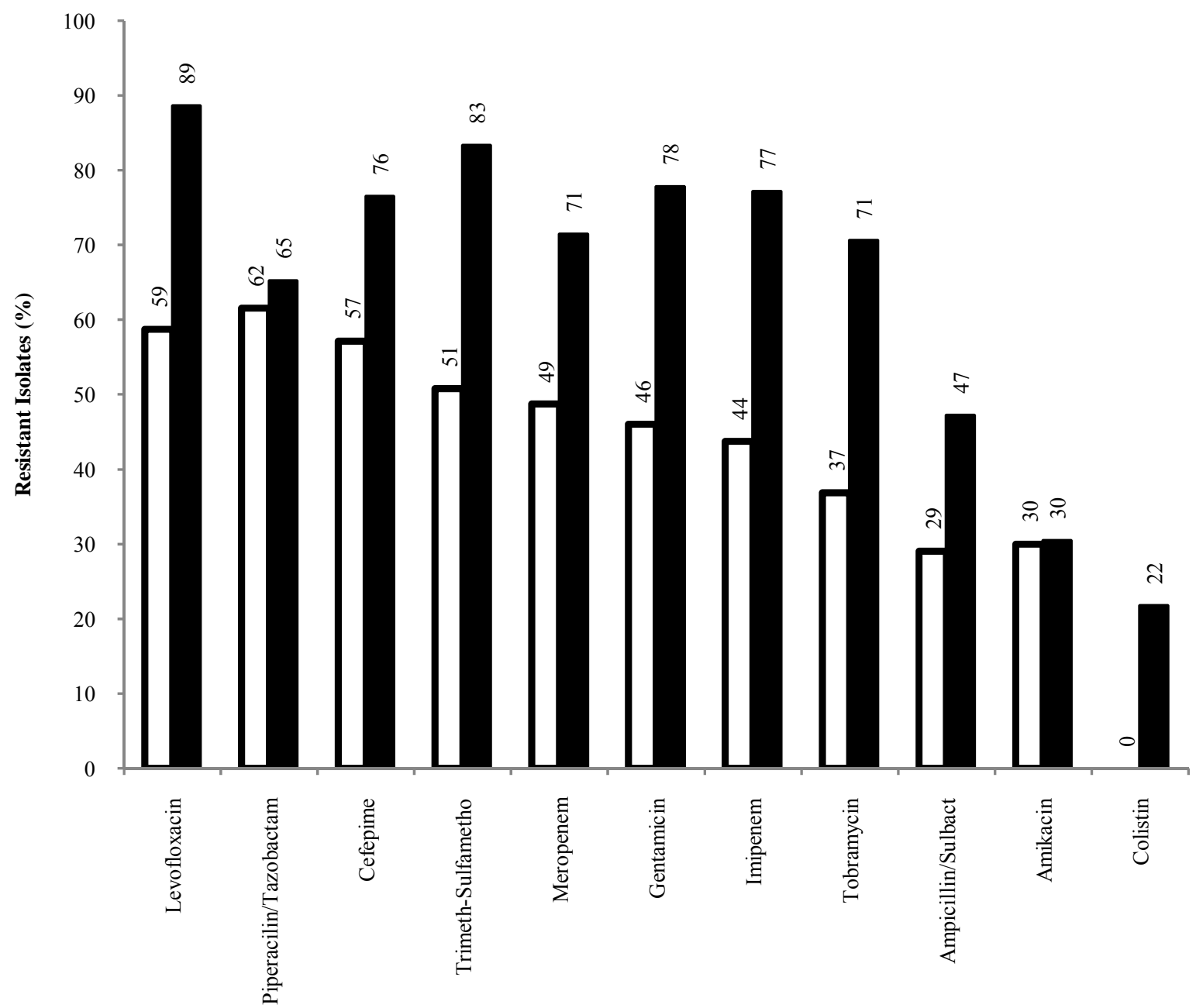

Antimicrobial agents

Figure 1. A comparative antibiogram of the first and repeat (second) isolates (white bar, first isolates, $n=64$; black bar, repeat isolate, $n=36)$.

Table 2. Risk factor analysis for carbapenem*.

\begin{tabular}{ccccccc}
\hline Risk Factor & Resistant & Susceptible & OR & CI & RR & P value \\
\hline Prior hospitalization & 43 & 25 & 5.56 & $2.70-11.47$ & 3.58 & $<0.05$ \\
Less than 1 month of hospitalization & 43 & 38 & 3.58 & $1.78-7.20$ & 2.8 & $<0.05$ \\
Polymicrobial infection & 35 & 22 & 2.16 & $1.14-4.1$ & 1.75 & $<0.05$ \\
Ventilator & 36 & 27 & 1.9 & $1.01-3.52$ & 1.65 & $<0.05$ \\
Sputum & 26 & 22 & 0.896 & $0.49-1.63$ & 0.91 & $>0.05$ \\
Diabetes & 19 & 11 & 0.415 & $0.22-0.79$ & 0.52 & $<0.05$ \\
\hline
\end{tabular}

${ }^{*}$ Carbapenem here includes imipenem and meropenem; and $p$ values were similar on both cases.

imipenem and meropenem. A significance of risk factors for both these agents are similar $(P<0.05)$, and separate data is not shown.

A comparative study of the risk factors associated with first and repeat isolates for carbapenem-resistance was performed (Table 3). Association of the development of resistance with certain risk factors was statistically significant for repeat isolates. Although ventilator use was 
Table 3. First and repeat isolate comparison of risk factors for carbapenem*.

\begin{tabular}{ccccccccc}
\hline \multirow{2}{*}{ Risk Factor } & \multicolumn{4}{c}{ First Isolates } & \multicolumn{3}{c}{ Repeat Isolates } \\
\cline { 2 - 10 } & OR & $\mathbf{9 5 \% ~ C I ~}$ & RR & P value & OR & $\mathbf{9 5 \% ~ C I ~}$ & RR & P value \\
\hline Ventilator & 1 & $0.45-2.23$ & 1 & $>0.05$ & 5.37 & $1.94-14.85$ & 4.4 & $<0.05$ \\
Diabetes & 1.18 & $0.53-2.63$ & 1.15 & $>0.05$ & 4.17 & $1.60-10.86$ & 3.5 & $<0.05$ \\
Surgery & NA & NA & NA & NA & 2.7 & $1.12-6.51$ & 2.37 & $<0.05$ \\
\hline
\end{tabular}

${ }^{*}$ Carbapenem here includes imipenem and meropenem, and $p$ values were similar in both cases.

not a significant factor for all isolates when considered together, it significantly increased the odds of resistance for repeat isolates for carbapenem $(\mathrm{OR}=5.37, \mathrm{CI}=1.94$ - 14.85; $P \leq 0.05)$. Diabetes, which was not seen as a major factor for overall development of resistance, was a risk factor for resistance development when studied repeat isolate from the specimen. This observation was evident from the increase in the odds ratio and statistical significance of the results $(\mathrm{OR}=4.17, \mathrm{CI}=1.6-10.86 ; P$ $\leq 0.05)$. The risk factor was considered only when it was applied to repeat isolates. Invasive procedures in the hospital were considered for their association with the resistant isolates. For the purpose of this study, debridement, biopsies, and major surgeries were considered as part of this category. Invasive procedures were an important risk factor for the development of resistance in repeat isolates.

\section{Discussion}

The findings demonstrate the occurrence of Acinetobacter infections in an urban hospital setting from the United States. Analysis of the clinical data provided information about the sources of the infections, with predominant risk factors associated with identified Acinetobacter infections. Age has been discussed as a major factor associated with the acquisition of Acinetobacter [23]. Although our sample size is smaller, a recent report on the analysis of 55,000 US surveillance samples indicates that people of working age have a higher risk of developing $A$. baumannii than do elderly people [24]. Men had a relatively higher risk of developing this infection. The higher occurrence of $A$. baumannii infection in working men could not be explained, but similar findings have been reported from other countries [25]. Duration of hospitalization is an important factor associated with nosocomial infections and hospital outbreaks. In the present study, shorter stay ( $>1$ week but $<1$ month) in the hospital contributed more to the incidence of infection. Prior and prolonged hospitalization and antibiotic therapy are established risk factors [10,18,26,27]. Therefore, the finding is difficult to explain. The possibilities, such as community acquisition or health care provider-associated dissemination, remain to be demonstrated. Sputum was a major source of $A$. baumannii, and the organism was associated with ventilator-associated pneumonia [28] and may indicate respiratory colonization and subsequent invasion [29]. A. baumannii was also isolated in conjunction with other nosocomial pathogens such as methicillin-resistant $S$. aureus and $P$. aeruginosa and denotes a dangerous combination of polymicrobial infections, offering more collective resistance.

Multidrug-resistant $A$. baumannii has been reported worldwide. In the present study, the large-scale occurrence of MDR- A. baumannii indicates increased prevalence in urban US hospital. The carbapenem-resistant $A$. baumannii isolates are increasingly reported from other US hospitals too and narrows the therapeutic options to control these superbugs. The large number of MDR- $A$. baumannii isolates prompted us to determine and systematically analyze the associated risk factors. Common risk factors suspected in previous studies [30], such as patient's time on a ventilator, were confirmed. The biggest risk factor for developing carbapenem-resistant $A$. baumannii was prior hospitalization. This risk factor has also been reported by other group [5] and could be indicative of exposures in the hospital environment or of the use of antibiotics that leads to development of resistance. Samples from the patients on respiratory ventilators and from those with polymicrobial infections [31] also had high odds of developing carbapenem resistant $A$. baumannii and suggest that cross contamination and colonization of respiratory equipment could be a concern. We also observed many repeat isolates among the total isolates and risk factors associated with resistance among them. Separate risk analysis between first and repeat isolates indicated the association of certain risk factors that were not significant in the overall analysis of all samples. The risk of developing repeat isolate resistant to carbapenems increased significantly with ventilator use. Invasive procedures have been associated with the multidrug resistance [7]. Diabetes and prior surgery, which were not significantly associated with overall resistance, were major risk factors in developing resistance in a second (or repeat) isolate. In our studies, many elderly patients who were hospitalized had a sacral decubitus ulcer, and 
were subsequently found to be infected [32]. These patients had come for a debridement procedure. The majority of these patients were ventilated, but a clear correlation could not be established in the acquisition of $A$. baumannii. These patients are usually given broad-spectrum antibiotics to control infection and are sent back to their long-term care facility and may be subsequently contributing to dissemination of A. baumannii in such health care facilities and the community at large. Further follow-up is required in such cases to gain more insight. We did not analyzed patient outcome and therapy evaluation analysis, which are beyond the scope of the present study. These analyses will require a manual data mining as not all the documents are retrievable.

In conclusion, a multidrug resistance was observed in a majority of the isolates. The repeat isolates displayed an increased level of antibiotic resistance. The resistance against all carbapenems and colistin is rapidly increasing; and the resistance was double in many of the repeat (second) isolates compared to initial (first) isolates against colistin, the drug of choice for such infections. A combination therapy is strongly advisable against such infections to avoid the emergence of colistin-resistant phenotypes in the United States hospitals.

\section{Acknowledgements}

This research work was partly supported by intramural funds from Drexel University College of Medicine, and partly, by the grant from Merck \& Co. Authors thank Ms. Pamela Fried for critical input in the manuscript. The study has IRB approval from the Drexel University College of Medicine.

\section{REFERENCES}

[1] M. Turkoglu, E. Mirza, O. G. Tunccan, et al., "Acinetobacter baumannii Infection in Patients with Hematologic Malignancies in Intensive Care Unit: Risk Factors and Impact on Mortality," Journal of Critical Care, Vol. 26, No. 5, 2011, pp. 460-467. doi:10.1016/j.jcrc.2011.04.007

[2] M. Shanthi and U. Sekar, "Multi-Drug Resistant Pseudomonas aeruginosa and Acinetobacter baumannii Infections among Hospitalized Patients: Risk Factors and Outcomes," Journal of the Association of Physicians of India, Vol. 57, 2009, pp. 636-645.

[3] H. Wisplinghoff, T. Paulus, M. Lugenheim, et al., "Nosocomial Bloodstream Infections Due to Acinetobacter baumannii, Acinetobacter pittii and Acinetobacter nosocomialis in the United States," Journal of Infection, Vol. 64, No. 3, 2012, pp. 282-290. doi:10.1016/j.jinf.2011.12.008

[4] T. J. Whitman, S. S. Qasba, J. G. Timpone, et al., "Occupational Transmission of Acinetobacter baumannii from a United States Serviceman Wounded in Iraq to a Health Care Worker," Clinical Infectious Diseases, Vol. 47, No.

\section{4, 2008, pp. 439-443. doi:10.1086/589247}

[5] M. Dizbay, O. G. Tunccan, B. E. Sezer, et al., "Nosocomial Imipenem-Resistant Acinetobacter baumannii Infections: Epidemiology and Risk Factors," Scandanavian Journal of Infectious Diseases, Vol. 42, No. 10, 2010, pp. 741-746. doi:10.3109/00365548.2010.489568

[6] J. Gomez, E. Simarro, V. Banos, et al., "Six-Year Prospective Study of Risk and Prognostic Factors in Patients with Nosocomial Sepsis Caused by Acinetobacter baumannii," European Journal Clinical Microbiology and Infectious Diseases, Vol. 18, No. 5, 1999, pp. 358-361. doi:10.1007/PL00015019

[7] J. Y. Jung, M. S. Park, S. E. Kim, et al., "Risk Factors for Multi-Drug Resistant Acinetobacter baumannii Bacteremia in Patients with Colonization in the Intensive Care Unit," BMC Infectious Diseases, Vol. 10, 2010, p. 228. doi:10.1186/1471-2334-10-228

[8] S. Yang, H. J. Yoon and M. R. Ki, "Risk Factors for Mortality in Acinetobacter bacteremia," Brazilianl Journal of Infectious Diseases, Vol. 15, No. 5, 2011, pp. 501502.

[9] C. G. Prates, A. F. Martins, S. V. Superti, et al., "Risk Factors for 30-Day Mortality in Patients with Carbapenem-Resistant Acinetobacter baumannii during an Outbreak in an Intensive Care Unit," Epidemiology and Infection, Vol. 139, No. 3, 2011, pp. 411-418. doi:10.1017/S0950268810001238

[10] S. G. Joshi, G. M. Litake, M. G. Satpute, et al., "Clinical and Demographic Features of Infection Caused by Acinetobacter Species," Indian Journal of Medical Sciences, Vol. 60, No. 9, 2006, pp. 351-360. doi:10.4103/0019-5359.27219

[11] M. del Mar Tomas, M. Cartelle, S. Pertega, et al., "Hospital Outbreak Caused by a Carbapenem-Resistant Strain of Acinetobacter baumannii: Patient Prognosis and RiskFactors for Colonisation and Infection," Clinical Microbiology and Infection, Vol. 11, No. 7, 2005, pp. 540-546. doi:10.1111/j.1469-0691.2005.01184.x

[12] S. F. Beavers, D. B. Blossom, T. L. Wiemke, et al., "Comparison of Risk Factors for Recovery of Acinetobacter baumannii during Outbreaks at Two Kentucky Hospitals, 2006," Public Health Reports, Vol. 124, No. 6, 2009, pp. 868-874.

[13] J. M. Cisneros, J. Rodriguez-Bano, F. Fernandez-Cuenca, et al., "Risk-Factors for the Acquisition of ImipenemResistant Acinetobacter baumannii in Spain: A Nationwide Study," Clinical Microbiology and Infection, Vol. 11, No. 11, 2005, pp. 874-879. doi:10.1111/j.1469-0691.2005.01256.x

[14] Y. S. Park, H. Lee, K. S. Lee, et al., "Extensively DrugResistant Acinetobacter baumannii: Risk Factors for Acquisition and Prevalent OXA-Type Carbapenemases: A Multicentre Study," International Journal of Antimicrobial Agents, Vol. 36, No. 5, 2010, pp. 430-435. doi:10.1016/j.ijantimicag.2010.06.049

[15] Y. J. Kim, S. I. Kim, Y. R. Kim, et al., "CarbapenemResistant Acinetobacter baumannii: Diversity of Resistant Mechanisms and Risk Factors for Infection," Epidemiol- 
ogy and Infection, Vol. 140, No. 1, 2012, pp. 137-145. doi:10.1017/S0950268811000744

[16] J. Baraibar, H. Correa, D. Mariscal, et al., "Risk Factors for Infection by Acinetobacter baumannii in Intubated Patients with Nosocomial Pneumonia," Chest, Vol. 112, No. 4, 1997, pp. 1050-1054. doi:10.1378/chest.112.4.1050

[17] T. H. Wong, B. H. Tan, M. L. Ling, et al., "Multi-Resistant Acinetobacter baumannii on a Burns Unit: Clinical Risk Factors and Prognosis," Burns, Vol. 28, No. 4, 2002, pp. 349-357. doi:10.1016/S0305-4179(02)00012-8

[18] W. H. Sheng, C. H. Liao, T. L. Lauderdale, et al., "A Multicenter Study of Risk Factors and Outcome of Hospitalized Patients with Infections Due to CarbapenemResistant Acinetobacter baumannii," International Journal of Infectious Diseases, Vol. 14, No. 9, 2010, pp. 764-769. doi:10.1016/j.ijid.2010.02.2254

[19] M. M. D'Andrea, C. Venturelli, T. Giani, et al., "Persistent Carriage and Infection by Multidrug-Resistant Escherichia Coli ST405 Producing NDM-1 Carbapenemase: Report on the First Italian Cases," Journal of Clinical Microbiology, Vol. 49, No. 7, 2011, pp. 2755-2758. doi:10.1128/JCM.00016-11

[20] R. Jayaraman, "Hypermutation and Stress Adaptation in Bacteria," Journal of Genetics, Vol. 90, No. 2, 2011, pp. 383-391. doi:10.1007/s12041-011-0086-6

[21] J. D. Pitout, K. B. Laupland, D. L. Church, et al., "Virulence Factors of Escherichia Coli Isolates that Produce CTX-M-Type Extended-Spectrum Beta-Lactamases," Antimicrobial Agents and Chemotherapy, Vol. 49, No. 11, 2005, pp. 4667-4670. doi:10.1128/AAC.49.11.4667-4670.2005

[22] J. Rodriguez-Bano, J. M. Cisneros, F. Fernandez-Cuenca, et al., "Clinical Features and Epidemiology of Acinetobacter baumannii Colonization and Infection in Spanish Hospitals," Infect Control and Hospital Epidemiology, Vol. 25, No. 10, 2004, pp. 819-824. doi:10.1086/502302

[23] S. M. Poutanen, M. Louie and A. E. Simor, "Risk Factors, Clinical Features and Outcome of Acinetobacter bacteremia in Adults," European Journal of Clinical Microbiology and Infectious Diseases, Vol. 16, No. 10, 1997, pp. 737-740. doi:10.1007/BF01709254

[24] R. M. Mera, L. A. Miller, H. Amrine-Madsen, et al., "Acinetobacter baumannii 2002-2008: Increase of Car-
bapenem-Associated Multiclass Resistance in the United States," Microbial Drug Resistance, Vol. 16, No. 3, 2010, pp. 209-215. doi:10.1089/mdr.2010.0052

[25] A. Abbo, S. Navon-Venezia, O. Hammer-Muntz, et al., "Multidrug-Resistant Acinetobacter baumannii," Emerging Infectious Diseases, Vol. 11, No. 1, 2005, pp. 22-29. doi:10.3201/eid1101.040001

[26] G. Baran, A. Erbay, H. Bodur, et al., "Risk Factors for Nosocomial Imipenem-Resistant Acinetobacter baumannii Infections," International Journal of Infectious Diseases, Vol. 12, No. 1, 2008, pp. 16-21. doi:10.1016/j.ijid.2007.03.005

[27] N. Y. Lee, H. C. Lee, N. Y. Ko, et al., "Clinical and Economic Impact of Multidrug Resistance in Nosocomial Acinetobacter baumannii Bacteremia," Infection Control and Hospital Epidemiology, Vol. 28, No. 6, 2007, pp. 713-719. doi:10.1086/517954

[28] J. Medina, C. Formento, J. Pontet, et al., "Prospective Study of Risk Factors for Ventilator-Associated Pneumonia Caused by Acinetobacter Species," Journal of Critical Care, Vol. 22, No. 1, 2007, pp. 18-26. doi:10.1016/j.jcrc.2006.06.010

[29] A. Martinez-Pellus, J. Ruiz Gomez, F. Jaime Sanchez, et al., "[Incidence of Colonization and Infection by Acinetobacter baumannii in an Endemic Seting (ICU). Analysis of Risk Factors by Means of a Surveillance Study]," Enfermedades Infecciosas y Microbiologia Clinica, Vol. 20, No. 5, 2002, pp. 194-199.

[30] H. C. Chang, Y. C. Chen, M. C. Lin, et al., "Mortality Risk Factors in Patients with Acinetobacter baumannii Ventilator: Associated Pneumonia," Journal of the Formosan Medical Association, Vol. 110, No. 9, 2011, pp. 564-571. doi:10.1016/j.jfma.2011.07.004

[31] P. A. Tilley and F. J. Roberts, "Bacteremia with Acinetobacter Species: Risk Factors and Prognosis in Different Clinical Settings," Clinical Infectious Diseases, Vol. 18, No. 6, 1994, pp. 896-900. doi:10.1093/clinids/18.6.896

[32] J. Koprnova, I. Svetlansky, R. Babel'a, et al., "Prospective Study of Antibacterial Susceptibility, Risk Factors and Outcome of 157 Episodes of Acinetobacter baumannii Bacteremia in 1999 in Slovakia," Scandanavian Journal of Infectious Diseases, Vol. 33, No. 12, 2001, pp. 891-895. doi:10.1080/00365540110076688

MIC: Minimum inhibitory concentration;

OR: Odds ratio;

RR: Relative risk;

CI: Confidence interval.
MRSA: Methicillin-resistant Staphylococcus aureus; MDR: Multi drug resistance; 